\title{
SUBJECTIVE WELL-BEING AND ITS IMPACT ON CANCER- RELATED FATIGUE IN PATIENTS UNDERGOING RADIOTHERAPY AND LONG-TERM SURVIVORS
}

\author{
R. Strebkova \\ Department of "Medical Psychology and Foreign Languages", Medical Faculty, \\ Trakia University - Stara Zagora, Bulgaria
}

\begin{abstract}
Objective: This study examines the profile of subjective well-being among 33 patients undergoing radiotherapy at the time of the study and 30 long-term survivors for over five years. A subject of the study is also the relation between the aspects of subjective well-being (life satisfaction, positive and negative affect) and the separate dimensions of fatigue and an analysis has been made of their influence on the burden of fatigue among the examined people. Materials and Methods: A total of 66 cancer patients were enrolled in the study - 33 patients undergoing radiotherapy and 30 long-term survivors. Multidimensional questionnaire on fatigue MFI-20 and Scale for subjective well-being (SWLS) were used to assess Cancer-related fatigue (CRF) and Subjective well-being in patients during radiation-treatment and in long-term survivors. Data were analyzed using the Spearman Correlation analysis and t-test of Student. Linear regression analysis was performed to determine the predictors of fatigue score during radiation-treatment and long-term survivor cancer patients. Results: Of all the components of subjective wellbeing, only life satisfaction and the positive affect showed a substantial relation with the five dimensions of fatigue, namely: general fatigue, physical fatigue, reduced activity, reduced motivation, mental fatigue. The burden of the physical and general fatigue among patients undergoing radiotherapy was estimated primarily based on the grounds of life satisfaction, $(\beta=-0.53 ; p=0.03)$, respectively; $(\beta=-0.40 ; p=0.01)$, the negative affect (stress) was a major predictor of the burden of general fatigue among patients belonging to the same group ( $\beta=0.33 ; p=0.03$ ). The positive affect predicts to a large extent the burden of the general $(\beta=-0.54 ; p=0.02)$ and physical fatigue $(\beta=-0.36 ; p=0.04)$ for the group of the long-term survivors for over five years. Conclusions: In congruence with positive psychology, positive affect and life satisfaction, as aspects of subjective well-being, proved to be protective factors against the burden of fatigue. These results can guide the development of individually tailored interventions that may reduce the impact of fatigue on patients with cancer.
\end{abstract}

Key words: cancer-related fatigue, subjective well-being, patients undergoing radiotherapy, longterm survivors

Corresponding author: Raisa Strebkova, PhD, Department "Medical Psychology and Foreign Languages", Medical Faculty, Trakia University, 6002 Stara Zagora, Bulgaria, e-mail: raja1@abv.bg 


\section{INTRODUCTION}

T he symptoms of fatigue and exhaustion are some of the most common problems among patients with malignant diseases $[27,28,29$, 5] and survivors [1], which are related to the disease and its treatment. According to [14], fatigue may occur during the treatment, in each stage of the disease and may last for months and even years after the completion of the treatment [13]. The most commonly used definition for Cancer-related fatigue (CRF) is found in the National Comprehensive Cancer Network's (NCCN) CRF evidence-based guidelines, where CRF is defined as a distressing persistent subjective sense of physical, emotional, and/or cognitive tiredness or exhaustion related to cancer or cancer treatment that is not proportional to recent activity that interferes with usual functioning [21].

Depending on the methods used for assessing fatigue and the characteristics of the groups of examined patients: localization of the process, stage of the disease, type of the disease [2] and others, its spread among patients with oncological diseases described in scientific literature varies widely from 14 to $99 \%$ $[20,26]$. During the stage of diagnosing, up to $40 \%$ of the patients report on the existence of fatigue [8]. During the active treatment, the indicated values of its spread range from $30 \%$ to $90 \%$ [25, 34]. Fatigue is extremely common among patients undergoing a cytotoxic chemotherapy, radiotherapy, bone marrow transplantation, treatment with biological response modifiers, including $\alpha$-interferon and interleukins [23, $16,12,15]$.

In 2007, Morrow et al. reported that for most patients with cancer, fatigue is more distressing and has a greater impact than other cancer-related symptoms such as pain, depression, and nausea. According to other authors [3,4], sustainable fatigue has a negative impact on the quality of the life of the affected patients, reducing their capacity to fully participate in various personal, professional and social activities and roles which make their life meaningful and impedes the implementation of typical cognitive tasks [7]. This impact is magnified by the increasing life expectancy of people with cancer and by the persistence of CRF for months or even years after the end of cancer treatment [19], but despite this, fatigue is rarely discussed by the patients and their oncologists [33]. The substantial negative effect on the quality of life, the physical and psycho-social well-being and the ability to perform the everyday activities of the affected patients [24], necessitates determining the phenomenon of fatigue as one of the main priorities in the care for patients with oncological diseases. In addition to this, as a subjective, complex and multifactor syndrome, fatigue can be better understood by including the patient's viewpoint.

Advances in diagnosis, intervention and treatment led to better prognosis for the oncology patients and to a stronger focus on patients' quality of life [18, 30], an important element of which are the mental health and the subjective well-being [22]. Subjective wellbeing is an important determinant of the "quality of life", which is now being considered as an endpoint in clinical trials [32]. Therefore, quality of life, subjective well-being are factors to be considered when choosing the best treatment.

Subjective well-being is based on the internal perspective of the person and, thus, puts the person's own concept on his/her life to the fore. It can be defined as a set of cognitive and affective assessments of the person of the life he/she is leading [22]. The structure of subjective well-being [11], which falls within the scope of the hedonistic perspective of happiness [17], includes three main components - life satisfaction, a positive affect and a negative affect. Each of these three main aspects can be disrupted. The positive affect is related to positive feelings and pleasant emotions like joy, happiness, pride and more. Negative affect refers to negative feelings and unpleasant emotions such as guilt, shame, anger, anxiety [10]. Subjective well-being can be assessed on a global level and also as a sum of the assessment of the separate aspects of life: satisfaction, positive affect, negative affect [9].

This study examines the profile of subjective wellbeing among patients undergoing radiotherapy at the time of the study and also long-term survivors for over five years. It studies the relation between the aspects of subjective well-being and the separate dimensions of fatigue and analyses their effect on the burden of fatigue among the examined people.

\section{MATERIAL AND METHODS}

\section{Study population}

Sixty-six (66) people with a confirmed oncological diagnosis were included in the study, 36 of whom were undergoing radiotherapy at the time of the study. Thirty (30) of the examined people have undergone radiotherapy and were presently long-term survivors who have lived over five years after being diagnosed with the disease. The examined people were aged 40-80. The average age of the patients in the examined group was mean $58.4(S D=10.8)$ years of age.

The examined people were divided into the following groups: 
1) patients currently undergoing radiotherapy $(n=36)$

2) people who have lived over 5 years from the moment they were diagnosed to the time the study was initiated $(n=30)$

Demographic data: the examined people were divided into the following groups: based on age: $40-50$ years of age $-18 ; 51-64$ years of age -29 and $65-80$ years of age - 19; based on marital status: 42 married, 4 unmarried, 6 divorced, 14 widowed; based on the place of residence: 37 in a city, 16 in a town, 13 in a village; based on education: 6 primary education, 32 secondary education, 28 university education; based on employment: employed - 37, unemployed -5 , retired -24 ; based on their incomes (at the discretion of the examined people): minimum -16 , good -46 , very good -4 .

Medical data: Groups of examined people based on the diagnosis: mammary gland carcinoma radical mastectomy (complete removal of the breast) (n = 23), mammary gland carcinoma quadrantectomy (removal of part of the breast) $(n=22)$, cervical carcinoma $(n=5)$, colorectal carcinoma $(n=5)$; other localizations $(n=11)$; based on the stage of the disease: I-II stage - 52, III-IV stage - 14; based on the treatment: surgical -60 have been operated on, 6 have not been operated on; chemotherapy - 32 have undergone chemotherapy, and 16 people to whom it has not been prescribed, 18 people to whom a hormone therapy has been prescribed and they are currently applying it; radiotherapy -36 people were undergoing radiotherapy at the time of the study, 30 of the people have undergone radiotherapy and are presently long-term survivors who have lived over five years from the moment they were diagnosed to the time the study was initiated.

\section{Methods/Measures}

The study was conducted on the territory of the Complex Oncological Centers in the city of Stara Zagora and the city of Burgas. After examining the documents, 85 patients were said to meet the inclusion criteria. All the patients were asked to take part in the study and 66 of them signed an informed agreement for participation in it.

1. A questionnaire on the socio-demographic features, the diagnosis and data about the disease. It has been made of 12 clearly formulated questions and includes some socio-demographic and medical data about 66 examined people. The questions give information about the age, the sex, the settlement, the marital status, the level of education, the economic status of the examined people, the diagnosis, the year of diagnosing, the stage of the disease and the type of the conducted treatment.
2. MFI-20-Multidimensional questionnaire on fatigue (Smets, Garssen, Bonke \& De Haes, 1996). A multidimensional tool for assessing fatigue. It has been additionally validated to be used for patients with cancer during the conducted study of 275 patients undergoing radiotherapy (Smets, Garssen, Cull \& de Haes, 1996.). The questionnaire (MFI-20) consists of five scales: general fatigue; physical fatigue; mental fatigue; decreased activity; decreased motivation, each of which contains four questions. It has been intended for patients with an oncological disease. The examined people give answers using a five-degree scale in a Likert format from 1 (yes, it is true) to 5 (no, it is not true). The results from each sub-scale range from 4 (lack of fatigue) to 20 (maximum fatigue).

3. Scale for evaluating subjective well-being (SWLS: Diener, Emmons, Larsen, \& Griffin, 1985). The scale of subjective well-being and life satisfaction has been established in order to measure the global cognitive assessment of life satisfaction, the feeling of happiness and the level of stress. The examined people indicate the extent to which they agree with each of the seven items of the scale, using the 4-degree Likert scale.

\section{Statistical Methods}

T-test of Student has been applied to compare the mean values of two independent extracts as well as a correlation analysis of Pearson to establish the statistically important correlations between the variables in the study. A regression analysis to assess the funcional dependence between two or more random quantities.

MFI-20 and SWLS subscores were calculated according to the instructions of the developers.

The data from the empirical study were statistically processed with the IBM SPSS Statistics, Version 20 software package. All results obtained were discussed at a statistical significance level of $P<0.05$.

\section{Area Descriptions}

In order to achieve the objectives, the following tasks have been set:

1. To study the relation between the aspects of subjective well-being and fatigue.

2. To compare the average values of subjective wellbeing among patients undergoing radiotherapy and survivors for over five years

3. To analyze the influence of the aspects of subjective well-being on the burden of the separate dimensions of fatigue among the two groups of examined people. 


\section{RESULTS}

For the purpose of examining the relation between the aspects of subjective well-being and the separate dimensions of fatigue, we made a correlation analysis (Table 1).

Interestingly, life satisfaction was in a significant negative correlation with the five dimensions of fatigue, namely: general fatigue $(r=-0.43, p<0.01)$, physical fatigue $(r=-0.46, p<0.01)$, reduced activity $(r=-0.45, p<0.01)$, reduced motivation $(r=-0.39$, $p<0.01)$ and mental fatigue $(r=-0.40, p<0.01)$. The same applies to the positive affect (experiencing the feeling of happiness) and the dimensions of general fatigue $(r=-0.43, p<0.01)$, physical fatigue $(r=-0.46, p<0.01)$, reduced activity $(r=-0.46, p<$ $0.01)$, reduced motivation $(r=-0.30, p<0.05)$ and mental fatigue $(r=-0.31, p<0.05)$. The positive affect was in a statistically significant correlation with life satisfaction $(r=0.62, p<0.01)$. It must be noted that the negative affect (stress) did not correlate sig- nificantly with any of the dimensions of fatigue and the positive aspects of subjective well-being, namely: life satisfaction and the experiencing of happiness of the examined patients.

When comparing the level of subjective well-being of the two groups of examined patients using Student's t-test, the measured levels of all the positive aspects of subjective well-being (life satisfaction, the feeling of happiness, family and job satisfaction) and the negative aspects in both groups are low. Significant differences were established only with reference to family relations satisfaction. The patients undergoing radiotherapy shared that they have a much higher level of family satisfaction compared to the group of survivors for over five years $(t=2.09 ; p=0.04<0.05)$ (Fig. 1).

In order to outline the differences between the two groups of examined people regarding the substantial predictors of each of the dimensions of fatigue among them, a regression analysis was used to study the effect of the separate aspects of well-being on the burden of fatigue.

Table 1. Relationship between aspects of subjective well-being and individual dimensions of fatigue.

\begin{tabular}{|l|c|c|c|c|c|c|c|c|}
\hline & $\mathbf{1}$ & $\mathbf{2}$ & $\mathbf{3}$ & $\mathbf{4}$ & $\mathbf{5}$ & $\mathbf{6}$ & $\mathbf{7}$ & $\mathbf{8}$ \\
\hline 1. General fatigue & 1 & $0.871^{* *}$ & $0.690^{* *}$ & $0.488^{* *}$ & $0.520^{* *}$ & $-0.430^{* *}$ & $-0.431^{* *}$ & .175 \\
\hline 2. Physical fatigue & & 1 & $0.790^{* *}$ & $0.571^{* *}$ & $0.561^{* *}$ & $-0.469^{* *}$ & $-0.468^{* *}$ & .208 \\
\hline 3. Decreased activity & & & 1 & $0.692^{* *}$ & $0.510^{* *}$ & $-0.451^{* *}$ & $-0.466^{* *}$ & .210 \\
\hline 4. Decreased motivation & & & & 1 & $0.655^{* *}$ & $-0.369^{* *}$ & $-0.301^{*}$ & .179 \\
\hline 5. Mental fatigue & & & & & 1 & $-0.409^{* *}$ & $-0.310^{*}$ & .210 \\
\hline 6. Life satisfaction & & & & & & 1 & $0.628^{* *}$ & -.020 \\
\hline 7. Positive affect & & & & & & & & -.025 \\
\hline 8. Negative affect. & & & & & & & & 1 \\
\hline
\end{tabular}

Note: the data were analyzed with Pearson linear correlation coefficient; the correlation is statistically significant: ${ }^{* *} p<0.01 ;{ }^{*} p<0.05$

\section{Family satisfaction}

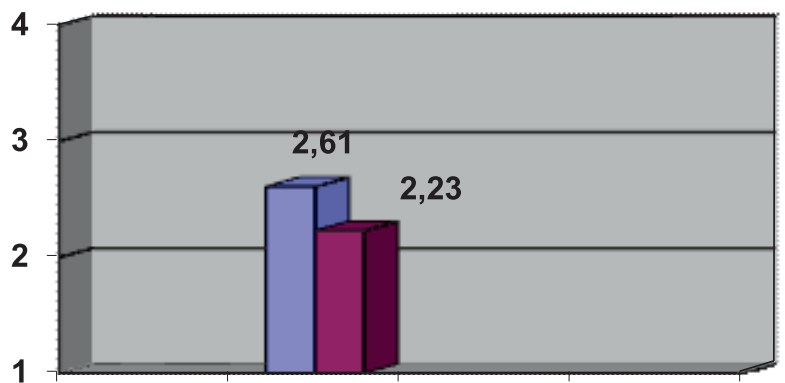

$\square$ patients undergoing radiotherapy

口long-term survivors

Fig. 1. Mean family satisfaction values for the two currently studied radiotherapy patients and survivors over five years 
In the group of those undergoing radiotherapy, the conducted regression analysis showed a model of two independent variables (Table 2), namely: Life satisfaction - it explains $32.4 \%$ of the variation of the dependent variable of physical fatigue and $24.3 \%$ of the changes in the burden of general fatigue. The negative affect (stress) can explain $23.1 \%$ of the changes in the burden of the dimension of general fatigue. Life satisfaction predicts negatively and substantially $(\beta=-0.53 ; p$ $=0.03<0.05)$ physical fatigue and serves as its most significant predictor. General fatigue is predicted negatively and substantially by life satisfaction $(\beta=-0.40 ; p$ $=0.01<0.05$ ) as well as positively and substantially by the negative affect (stress) $(\beta=0.33 ; p=0.03<0.05$ ).

In the group of patients who have survived for more than five years, a pattern of one statistically significant factor was established - the positive affect with a negative and substantial influence on the burden of general fatigue $(\beta=-0.54 ; p=0.02<0.05)$ and physical fatigue $(\beta=-0.36 ; p=0.04<0.05)$.

Table 2. Regression analysis of the influence of aspects of subjective well-being on the severity of fatigue in the groups of patients studied

\begin{tabular}{|l|c|c|c|c|}
\hline Physical fatigue & Beta & $\mathbf{P}$ & $\mathbf{F}$ & $\mathbf{R}^{\mathbf{2}}$ \\
\hline $\begin{array}{l}\text { Currently undergoing } \\
\text { radiation treatment }\end{array}$ & & & & \\
\hline Life satisfaction & -0.53 & $p=0.03$ & 5.10 & $0.324(32.4 \%)$ \\
\hline $\begin{array}{l}\text { Survived for over five } \\
\text { years }\end{array}$ & & & & \\
\hline Positive affect & -0.36 & $p=0.04$ & 4.18 & $0.291(29.1 \%)$ \\
\hline
\end{tabular}

\begin{tabular}{|l|c|c|c|c|}
\hline General fatigue & Beta & $\mathbf{P}$ & $\mathbf{F}$ & $\mathbf{R}^{\mathbf{2}}$ \\
\hline $\begin{array}{l}\text { Currently undergoing } \\
\text { radiation treatment }\end{array}$ & & & & \\
\hline Life satisfaction & -0.40 & $\mathrm{p}=0.01$ & 6.72 & $0.243(24.3 \%)$ \\
\hline Negative affect & 0.33 & $\mathrm{p}=0.03$ & 4.75 & $0.231(23.1 \%)$ \\
\hline $\begin{array}{l}\text { Survived for over five } \\
\text { years }\end{array}$ & & & & \\
\hline Positive affect & -0.54 & $\mathrm{p}=0.02$ & 11.7 & $0.271(27.1 \%)$ \\
\hline
\end{tabular}

\section{DISCUSSION}

When examining the profile of subjective well-being, studying the relations between its aspects and the separate dimensions of fatigue, the established correlations are complex. The correlation analyses have revealed that the dimensions of fatigue are related to the positive aspects of subjective well-being but none of the dimensions of fatigue correlates with the negative aspect of subjective well-being, which is the stress. This leads us to the conclusion that the experiencing of pleasant emotions, happiness, a low level of the negative moods and a high level of life satisfaction can act as buffers against the burden of fatigue.
It was established that the levels of all positive aspects of subjective well-being (life satisfaction, a feeling of happiness, family and job satisfaction) and the negative aspects among both groups are low.

Based on the conducted analyses, we can summarize that the patients undergoing radiotherapy share that they have a significantly higher level of family satisfaction compared to the group of the survivors for over five years. The results obtained from the study contradict the resuts from the study of [6]. The result obtained in this study can be discussed in relation to the greater connectedness of these patients with their families, owing to their explicit need of care, understanding and support, as they are in a period of active treatment accompanied by a number of side effects.

The applied regression analysis of the influence of the separate aspects of well-being on the burden of fatigue has established that life satisfaction significantly predicts the burden of physical and general fatigue among patients undergoing radiotherapy and the negative effect (the level of stress) is a significant predictor of the general fatigue of patients from the same group.

For the group of the long-term survivors, it has been established that the positive affect is the most significant predictor of the burden of general fatigue and physical fatigue. The positive emotions (positive affect) probably acquire a meaning to the patients with malignant diseases at a later stage of the trajectory of the disease, after they have managed to adapt to the disease itself, have established strategies for coping and have developed an ability to control the negative emotions related to the disease. The data obtained from the conducted analysis give ideas for the direction of the therapeutic interventions in the different stages of the disease.

The conducted study has shown the importance of the examination of the dimensions of subjective wellbeing as determinants of fatigue among the studied patients with oncological diseases.

The analysis of these mutual influences provides a material that gives ideas about how it is possible, by stimulating the positive functioning of the sick person, to influence the intensity of suffering and, in the end, the quality of life. In this sense, it is important to develop and increase the resources and the capacity of the person to have a positive view on life and experience positive emotions which can be used as a powerful therapeutic means for treating complex problems, such as the fatigue among patients with oncological diseases.

\section{CONCLUSION}

The burden of fatigue among the examined groups of patients with malignant diseases seems to be large- 
ly dependent on the separate aspects of subjective well-being (happiness and life satisfaction). Considering the data obtained from the study, we can regard life satisfaction as a buffer against the escalation of general and physical fatigue.

The disease gives the person some opportunities which are to be discussed with the patients in the context of its relation to suffering and emotional losses. One of these opportunities is related to the approximation between the family members and the chance they have to spend plenty of satisfying and rewarding moments together. This fact is supported by the present study because the patients undergoing radiotherapy report of a much higher level of subjective well-being compared to the group of the long-term survivors.

For the patients currently undergoing radiotherapy, it is important to reduce the level of stress in order to survive lower levels of fatigue. Unlike them, for the long-term survivors it is more important to have more positive emotions, because the positive aspect is the most significant predictor of the burden of general fatigue and physical fatigue among them.

Disclosure summary: The authors have nothing to disclose.

\section{REFERENCES}

1. Ahlberg K, Ekman T, Gaston-Johansson F, et al. Assessment and management of cancer-related fatigue in adults. Lancet, 2003 362: 640-650.

2. Berger AM, Lockhart K, Agrawal S. Variability of Patterns of Fatigue and Quality of Life Over Time Based on Different Breast Cancer Adjuvant Chemotherapy Regimens. Oncology Nursing Forum, 2009 [s. I.], v. 36, n. 5, 563-570.

3. Cella D. The Functional Assessment of Cancer Therapy-Anemia (FACT-An) Scale: A new tool for the assessment of outcomes in cancer anemia and fatigue. Semin Hemato, 1997, I 34:(suppl 2), 13-19.

4. Cella D, Tulsky D, Gray G, et al. The functional assessment of cancer therapy scale: Development and validation of the general measure. J Clin Oncol, 1993, 11: 570-579.

5. Cella D, Davis K, Breitbart W, Curt G. Cancer-related fatigue: prevalence of proposed diagnostic criteria in a United States sample of cancer survivors.J Clin Oncol., 2001, 19: 3385-91.

6. Chandra PS, Chaturvedi SK, Channabasavanna SM et al. „Psychological Well-Being among Cancer Patients Receiving Radiotherapy: A Prospective Study." Quality of Life Research 7, no. 6, 1998, 495-500. www.jstor.org/stable/4034803.

7. Curt GA, Breitbart W, Cella D et al. Impact of cancer-related fatigue on the lives of patients: new findings from the Fatigue Coalition. Oncologist, 2000, 5, 353-360; 553-560.

8. de Vries U, Reif K, Peterman F. Tumorbedingte Fatigue und ihre psychosozialen Belastungen November 2011, 85-92.

9. Diener E, Lucas, RE, Oishi,S. Subjective well-being: The science of happiness and life satisfaction. In C. R. Snyder \& S. J. Lopez (Eds.), Handbook of positive psychology, 2002, 63-73, New York: Oxford University Press.

10. Diener E, Gohm C, Suh E, Oishi S. Similarity of the relations between marital status and subjective well-being across cultures. Journal of Cross-Cultural Psychology, 1998, 31 (4), 419-436.

11. Diener E, Emmons RA, Larsen RJ et al. The Satisfaction with Life Scale. Journal of Personality Assessment, 1985, 49, 71-75.
12. Hann DM, Garovoy N, Finkelstein B, et al. Fatigue and quality of life in breast cancer patients undergoing autologous stem cell transplantation: A longitudinal comparative study. Journal of Pain and Symptom Managemen, 1999, 17:311-19 [PubMed].

13. Hofman M, Ryan JL, Figueroa-Moseley C D et al. Cancerrelated fatigue: the scale of the problem. Oncologist, 2007, 12 Suppl 1 4-10.

14. Horneber M., Fischer I., Dimeo F., Ulrich Ruffer J. Tumorassoziierte Fatigue Epidemiologie, Pathogenese, Diagnostik und Therapie Deutsches Ärzteblatt, 2012, Jg. 109,| Heft, 2. März s.161-171.

15. Jacobsen PB, Stein K. Is Fatigue a Long-term Side Effect of Breast Cancer Treatment? Cancer Control, 1999 6(3):256-63.

16. Irvine D, Vincent L, Graydon JE, et al. The prevalence and correlates of fatigue in patients receiving treatment with chemotherapy and radiotherapy. A comparison with the fatigue experienced by healthy individuals. Cancer Nurs., 1994, 17:367-378.

17. Kahneman D, Diener E, Schwarz N. (eds). Well-Being: The Foundations of Hedonic Psychology (Russel Sage Foundation New York), 1999.

18. Monteiro-Grillo I, Marques-Vidal P, Jorge M. Psychosocial effect of mastectomy versus conservative surgery in patients with early breast cancer. Clinical and Translational Oncology, 2005, 7:499-503

19. Morrow GR. Cancer-related fatigue: Causes, consequences, and management. Oncologist, 2007, 12 (1, Suppl.), 1-3. doi: 10.1634/ theoncologist.12-S1-1

20. National Comprehensive Cancer Network. Clinical practice guidelines in oncology. Cancer related fatigue. Online unter http://www.nccn. org. Zugegriffen 01. Juni (2011).

21. National Comprehensive Cancer Network. NCCN Clinical Practice Guidelines in Oncology: Cancer-Related Fatigue. (2010) Retrieved from http://www.nccn.org.

22. Petkova M. Subjective well-being and health, KOTA, Stara Zagora, 2002, 24-32.

23. Piper BF, Rieger PT, Brophy L et al. Recent advances in the management of biotherapy-related side effects: fatigue. Oncol Nurs Forum, 1989, 16(suppl 6):27.

24. Portenoy RK, Itri LM. Cancer-related fatigue: guidelines for evaluation and management. Oncologist, 1999, 4 (1), 1-10.

25. Prue G, Rankin J, Allen J et al. Cancer-related fatigue: a critical appraisal. Eur. J. Cancer, 2006, 42:846-863.

26. Servaes $P$, Gielissen MF, Verhagen $S$, et al. The course of severe fatigue in disease-free breast cancer patients: a longitudinal study. Psychooncology, 2007, 16: 787-95.

27. Servaes P, Verhagen C, Bleijenberg G. Fatigue in cancer patients during and after treatment: prevalence, correlates and interventions. Eur J Cancer, 2002a, Jan, 38(1):27-43.

28. Servaes P, Verhagen CA, Bleijenberg G. Relations between fatigue, neuropsychological functioning, and physical activity after treatment for breast carcinoma: daily self-report and objective behaviour. Cancer, 2002b Nov 1; 95(9):2017-26.

29. Servaes P, Verhagen S, Bleijenberg G. Determinants of chronic fatigue in disease-free breast cancer patients: a cross-sectional study. Ann Oncol., 2002c, Apr; 13(4):589-98.

30. Shimozuma K, Imai H, Kuroi K et al. Recent topics of health outcomes research in oncology. Breast Cancer, 2007, 14:60-5.

31. Smets EM, Garssen B, Cull A, et al. Application of the multidimensional fatigue inventory (MFI-20) in cancer patients receiving radiotherapy. Br J Cancer, 1996, 73(2): 241-245.

32. Spilker B. Quality of life assessments in clinical trials. Raven Press, New York. Google Scholar, 1990.

33. Vogelzang NJ, Breitbart W, Cella D, et al. Patient, caregiver, and oncologist perceptions of cancer-related fatigue: results of a tripart assessment survey. The Fatigue Coalition. Semin Hematol., 1997, 34:4-12.

34. Wagner LI, Cella D. Fatigue and cancer: Causes, prevalence and treatment approaches. Br J Cancer, 2004, 91:822-828.

Received: 10 June 2020, Accepted: 1 August 2020 